\title{
OTIMIZAÇÃO UTILIZANDO A HEURÍSTICA DAS P-MEDIANAS PARA IDENTIFICAR LOCAIS PARA INSTALAÇÃO DE INSTITUTOS FEDERAIS NO ESTADO DE SANTA CATARINA
}

\author{
OPTIMIZATION USING THE P-MEDIAN HEURISTICS TO IDENTIFY LOCATIONS FOR FEDERAL INSTITUTES \\ FACILITIES IN THE STATE OF SANTA CATARINA
}

\section{iD Silvio Aparecido da Silva ${ }^{1}$ \\ iD Andréa Cristina Konrath ${ }^{2}$ \\ iD Antônio Cezar Bornia ${ }^{3}$}

${ }^{1}$ Doutorando do Programa de Pós-Graduação em Engenharia de Produção na Universidade Federal de Santa Catarina - UFSC. Mestrado em Métodos Numéricos em Engenharia. Florianópolis, Santa Catarina, Brasil.silvioest@gmail.com

2 Doutorado em Engenharia Mecânica pela Universidade Federal de Santa Catarina (2008).

Professora adjunta na Universidade Federal de Santa Catarina (UFSC), pertencendo ao Departamento de Informática e Estatística. Florianópolis, Santa Catarina, Brasil.andreack@gmail.com

${ }^{3}$ Doutorado em Engenharia de Produção pela Universidade Federal de Santa Catarina. professor titular da Universidade Federal de Santa Catarina, lotado no Departamento de Engenharia de Produção e Sistemas. Florianópolis, Santa Catarina, Brasil. cezar.bornia@gmail.com

Recebido em: 25 nov. 2019

Aprovado em: 13 fev. 2020

Cite como - American Psychological Association (APA)

Silva, S. A. da., Konrath, A. C., \& Bornia, A. C., (2021, jan./mar.). Otimização Utilizando a Heurística das P-Medianas para Identificar Locais para Instalação de Institutos Federais no Estado de Santa Catarina. Exacta, 19(1), 150-165. https://doi.org/10.5585/exactaep.v19n1.15926.
Resumo: O objetivo deste artigo é aplicar uma heurística para localização de pmedianas, no sentido de identificar os melhores locais para instalação de Institutos Federais no estado de Santa Catarina, de maneira a minimizar custos com transporte. Foram utilizados três cenários: o primeiro considera a população do estado de Santa Catarina, enquanto o segundo, o número de alunos que completaram o Ensino Fundamental e o Ensino Médio. Já o terceiro cenário considera a taxa de analfabetismo. A otimização foi feita por meio da heurística de Teitz e Bart das p-medianas. A heurística Teiz e Bart demonstrou ser uma excelente aproximação para o problema das p-medianas e apresentou um excelente resultado para tomada de decisão na administração pública.

Palavras-chave: Administração Pública. P-mediana. Heurística. Teiz e Bart.

Otimização.

Abstract: The purpose of this article is to apply a heuristic for the localization of $p$ medians, as a mean to identify the best locations for Federal Institutes in the state of Santa Catarina, in order to minimize transportation costs. Three scenarios were used: the first considers the population of Santa Catarina state, while the second, the number of students who have completed the primary school and the high school. The third scenario considers the illiteracy rate. The optimization was done through the heuristic of Teitz and Bart of the $p$-medians. The Teiz and Bart heuristic has proved to be an excellent approach for the $p$-median problem and presented outstanding results for decision-making by the public administration.

Keywords: Public Administration. P-median. Heuristic. Teitz and Bart. Optimization. 


\section{Introdução}

A verificação da localização de instalações é de suma importância para realizar uma boa gestão, seja ela no setor público ou privado. Se a localização for feita de forma adequada, esta trará o maior benefício social possível, maximizando o nível de serviço prestado (Daskin, 1995). Caso contrário, pode criar problemas de produtividade ou na prestação de serviços após sua execução (Randhawa \& West, 1995; Romero, 2006).

No problema de localização, deseja-se estabelecer os locais nos quais serão encontradas facilidades para satisfazer, da melhor maneira possível, um conjunto espacialmente distribuído de pontos de demanda. Conforme Pizzolato, Raupp e Alzamora (2012), a localização de facilidades pode ser aplicada em diversas áreas. Dentre os diversos estudos que utilizaram o problema de localização, pode-se destacar: localização de centros de distribuição (Froes et al., 2019; Calache \& Camargo, 2016); planejamento de tráfico rodoviário (Camara et al., 2016); unidades de saúde (Goudard et al., 2015); e unidades educacionais (Garcia, 2015). Tratando-se da área de educação, ela pode auxiliar na diminuição do tempo de locomoção dos alunos até a escola, na redução de gastos com transporte e em maior satisfação dos servidores (Garcia, 2015).

Diversas técnicas de modelagem são conhecidas como modelos de pesquisa operacional, que são utilizados para analisar sistemas reais, por meio de técnicas matemáticas, objetivando a determinação da melhor solução para o sistema representado.

Um modelo para localização de centralizadores pode ser formulado como um problema de $p$ medianas, no qual, definida a variável custo e a quantidade de $p$-medianas desejada, e assumindo a variável de decisão como binária, o modelo define a melhor configuração para sua localização em relação aos outros elementos analisados. Assim, o problema de $p$-medianas é um problema tradicional de localização, e tem o objetivo de minimizar o somatório das distâncias entre cada nó de demanda e a mediana mais próxima (Hakimi, 1964, 1965).

No que tange à área educacional, para a qual existem diferentes cenários que são considerados complexos de gerir, existe uma necessidade de utilizar métodos adequados e confiáveis (Garcia, 2015). Neste sentido, o objetivo principal deste trabalho é aplicar uma heurística para localização de $p$ medianas, no sentido de identificar os melhores locais para instalação de Institutos Federais no estado de Santa Catarina, de forma que atenda às localidades com o menor custo (distância) possível, dado que, para cada região, existe uma demanda diferente, o que impacta diretamente no custo de transporte, e uma variável selecionada, que atuará como peso para melhor decisão.

Deste modo, foram propostos três cenários para as diferentes demandas. O primeiro modelo considera a população, o segundo considera alunos formando-se no Ensino Fundamental mais os alunos formando-se no Ensino Médio, e o terceiro modelo que considera a quantidade de analfabetos. Para cada cenário, duas novas localidades foram solicitadas, ou seja, supondo que os Institutos tenham orçamento para abrir duas novas unidades, buscou-se identificar quais seriam as cidades escolhidas. 


\section{Desenvolvimento}

\subsection{Instituto Federal}

Os Institutos Federais de Educação, Ciência e Tecnologia foram criados por meio da Lei Federal no 11.892, de 29 de dezembro de 2008, que instituiu todos os Institutos Federais no Brasil, e têm como principais metas: a manutenção de pelo menos $50 \%$ de matrículas no ensino técnico de nível médio; manutenção de pelo menos $20 \%$ de matrículas em cursos de licenciaturas e de formação de professores; e o compromisso da oferta de curso do Programa Nacional de Integração da Educação Profissional com a Educação Básica, na Modalidade de Educação de Jovens e Adultos PROEJA (técnico e FIC), na perspectiva de promover a inclusão e atender a demanda regional. O estado de Santa Catarina possui dois Institutos Federais, denominados: Instituto Federal de Santa Catarina (IFSC) e o Instituto Federal Catarinense (IFC), os quais estão espalhados em diferentes cidades.

O IFSC é formado por 23 campi espalhados por todo o estado de Santa Catarina, com um total de 45.192 matrículas atendidas e um quadro funcional de 2.579 servidores entre professores e técnicos no ano de 2017 (Instituto Federal de Santa Catarina [IFSC], 2018). O IFC é formado por 15 campi, espalhados por todo o estado de Santa Catarina, com um total de 18.006 matrículas atendidas e um quadro funcional de 1.910 servidores entre professores e técnicos no ano de 2017 (Instituto Federal Catarinense [IFC], 2018).

\subsection{O Problema da p-Mediana}

Atualmente, os problemas de localização têm diversas aplicações, que vão da simples localização de um depósito, fábrica, escola ou posto policial, passando pela localização de antenas, centrais telefônicas, estações de tratamento de água, até a localização de roteadores em redes de computadores (Horner, 2009).

O problema da localização de $p$-medianas tem o objetivo de localizar $p$ facilidades, de tal forma que a distância entre estas $p$ instalações e os $n$ locais de demanda seja minimizada. Observa-se que não se trata de um caso emergencial, mas sim de minimização das somas das distâncias percorridas (Larson \& Odoni, 1981). A primeira formulação do problema de $p$-mediana é apresentada por Hakimi (1964). O problema das $p$-medianas formulado por Christofides (1975) consiste em um problema de programação inteira binária (PIB), da seguinte forma: 
Minimizar $Z=\sum_{i=1}^{n} \sum_{j=1}^{n} w_{i} c_{i j} x_{i j}$

sujeito à:

$\begin{array}{ll}\sum_{i=1}^{n} & x_{i j}=1,(j=1,2, \ldots, n) \\ \sum_{i=1}^{n} & x_{i i}=p,(i=1,2, \ldots, n)\end{array}$

$x_{i j} \geq x_{i i}$

$x_{i j} \in 0,1,(i=1,2, \ldots, n)(j=1,2, \ldots, n)$

Em que a variável custos (ou distâncias) é dada por $c_{i j}$, com $c_{i i}=0$ para todo $i \in n$; a variável $x_{i j}$ é definida como de alocação, com $x_{i j}=1$ se o nó $j$ é alocado à mediana $i$, e $x_{i j}=0$; caso contrário, $p$ é o número de medianas e $n$ é o número de nós; e para este modelo é considerada a variável de peso $w_{i}$, associado ao vértice $i$.

As restrições (2) e (4) garantem que cada nó $j$ é alocado a apenas um nó i, o qual deve ser uma mediana. A restrição (3) determina o número exato de medianas a ser localizado e a restrição (5) corresponde às condições de integralidade.

Mesmo com os avanços, na área de informática, dedicados à solução de problemas de programação matemática, esta formulação do problema das p-medianas é de difícil solução, necessitando procedimentos heurísticos para sua resolução. A heurística de Teitz e Bart (1968) tem apresentado bom desempenho para a resolução deste tipo de problema.

\subsection{Heurística de Teitz e Bart}

Goldbarg e Luna (2000) definem a heurística como: "uma técnica que busca alcançar uma boa solução utilizando um esforço computacional considerado aceitável, sendo capaz de garantir a viabilidade ou a otimalidade da solução encontrada ou, ainda em muitos casos, ambas, especialmente nas ocasiões em que essa busca partir de uma solução viável próximo ao ótimo". (Goldbarg \& Luna, 2000, p. 244)

A heurística das $p$-medianas de Teitz e Bart é baseada na substituição de vértices e seu objetivo é, a partir de uma solução inicial, melhorar o valor da função objetivo a cada iteração. A implementação computacional desta heurística é fácil e produz soluções satisfatórias para os problemas de localização de facilidades. Chama-se de número de transmissão do vértice $v_{i}$, representado por $\sigma\left(v_{i}\right)$, a soma das menores distâncias existentes entre o vértice $v_{i}$ e todos os outros vértices $v_{j}$ do grafo. Sendo n o número total de vértices do grafo, o número de transmissão $\sigma_{0}\left(v_{i}\right)$ é dado por: 
$\sigma_{0}\left(v_{i}\right)=\sum_{v_{j} \in V}^{n} \quad w_{i} d\left(v_{i}, v_{j}\right)$

Onde $d\left(v_{i}, v_{j}\right)$, é a menor distância entre os vértices $v_{i}$, e $v_{j}, w_{i}$ é o peso associado ao vértice $v_{i}$.

Escolhe-se, inicialmente, $p$ vértices para formar um conjunto $S$ inicial, o qual é considerado uma aproximação do conjunto $V$ das $p$-medianas. Sendo $V$ o conjunto de todos os vértices do grafo, verificase se qualquer vértice $v_{i}$ pertencente ao conjunto $V$-S pode-se substituir um vértice $v_{j}$ pertencente a $S$ e produzir um novo conjunto $S^{\prime}$, em que

$$
S^{\prime}=S \cup\left\{v_{i}\right\}-\left\{v_{j}\right\}
$$

para o qual o número de transmissão $\sigma\left(S^{\prime}\right)<\sigma(S)$. Se isto for possível, é feita a substituição de $v_{j}$ por $v_{i}$, e $S^{\prime}$ é uma nova aproximação para o conjunto $S$. O processo continua até obter-se um conjunto $S$, em que nenhuma substituição de vértice de $S$ por outro em $V$-S produz um número de transmissão menor.

Os passos do algoritmo são os seguintes:

Passo 1. Selecione um conjunto $S$, com $|S|=p$ para formar uma aproximação inicial para as $p$-medianas; Passo 2 . Rotule todos os vértices $v_{j}$ pertencente a $V$-S como não analisados;

Passo 3. Enquanto existirem vértices "não analisados" em V-S. Faça:

a) Selecione um vértice $v_{j}$ pertencente a $V$-S "não analisado", e calcule a redução $\Delta$ do número de transmissão, para todo $v_{j}$ pertencente $\mathrm{S} S$, tal que:

$\Delta_{i j}=\sigma(S)-\sigma\left(S \in\left\{v_{i}\right\}-\left\{v_{j}\right\}\right)$

b) Faça $\Delta_{i j 0}=\max \left[\Delta_{i j}\right]$;

c) Se $\Delta_{i j o}>0$, faça $S=S \in\left\{v_{i}\right\}-\left\{v_{j 0}\right\}$ e rotule $v_{j 0}$ como analisado;

d) Se $\Delta_{i j 0}>0$, rotule $v_{i}$ analisado.

Passo 4. Se durante a execução do Passo 3 houver algumas modificações no conjunto S, volte ao Passo

2. Caso contrário, pare e apresente o conjunto S como uma aproximação para a solução do problema das $p$-medianas.

\section{Material e método}

Este trabalho pode ser considerado uma pesquisa aplicada, conforme definido por Barros e Lehfeld (2000) e Gil (2017), pois tem a finalidade de desenvolver conhecimentos mediante a aplicação prática, bem como a resolução de problemas encontrados na realidade.

Os dados da localização dos Institutos Federais do estado de Santa Catariana foram coletados no site da própria instituição. Foram contabilizados 23 campi do IFSC, localizados em 22 cidades diferentes, e 15 campi do IFC, localizados em 15 cidades diferentes, totalizando 35 cidades com 
Institutos Federais em Santa Catarina.

A população residente por cidade do Estado, estimada no ano de 2015, foi obtida no site do IBGE com data de referência de 1 de julho de 2015, (Instituto Brasileiro de Geografia e Estatística [IBGE], 2015a). A população de alunos no último ano do Ensino Médio e alunos no último ano do Ensino Fundamental (formandos) foi obtida nos dados do censo educacional de 2012, realizado pelo Instituto Nacional de Estudos e Pesquisas Educacionais Anísio Teixeira [INEP] (2012). A taxa de analfabetismo por cidade foi calculada utilizando a taxa de alfabetização de pessoas de cinco anos ou mais, obtida na base de dados do censo de 2010 (IBGE, 2011).

O tratamento dos dados foi realizado no ambiente R (R Core Team, 2018), no qual foi realizada a produção dos mapas temáticos e as coordenadas, utilizando o pacote maptools (Bivand et al., 2018). Em seguida, foi calculada a distância euclidiana entre os centroides das cidades.

Para o cálculo das p-medianas, foi utilizado o pacote tbart de Brunsdon (2015), que utiliza o algoritmo de Teiz e Bart. Foram utilizadas as variáveis população, formandos e taxa de analfabetismo como pesos para cada cenário. Foram solicitadas duas novas p-medianas (localidades), as quais representam os possíveis locais de decisão de novas unidades dos Institutos Federais em Santa Catarina.

\section{Discussão da aplicação do problema das $p$-medianas}

O estado de Santa Catarina é formado por 295 municípios; assim, foram calculados 295 centroides das cidades do estado e atribuído o valor um para as cidades $i$ que já possuem campus dos Institutos Federais. Como avalia-se a necessidade de uma cidade receber um Instituto, no caso onde o Instituto Federal possui dois campi, foi atribuído um ponto apenas. Foram calculadas as distâncias euclidianas de um centroide a outro, gerando uma matriz de distância de dimensão 295 x 295, com a diagonal igual a zero.

O total de Institutos espalhados pelo Estado é de 35 unidades. Portanto, para aplicação do algoritmo de Teiz e Bart, foram atribuídos para a solução inicial os 35 pontos, mais a quantidade de pontos que se deseja localizar. Neste caso, a solução inicial do vértice $v_{i}$, onde os $i$ são as localidades já existentes dos Institutos Federais, é rotulada como analisada. Desta forma, resta analisar os pontos a serem localizados.

Para a variável de peso $w_{i}$, foram consideradas três possíveis variáveis diferentes para a demanda: o primeiro modelo considera a população estimada pelo IBGE no ano de 2015, o segundo considera alunos se formando no Ensino Fundamental e mais alunos se formando no Ensino Médio, e o terceiro modelo que considera a taxa de analfabetos

\subsection{Cenário 1 - População Estimada 2015}

O primeiro modelo considerou como o peso $w_{i}$ a estimativa da população de 2015. A projeção 
das populações é feita anualmente pelo IBGE e serve de base para o repasse de recursos do orçamento aos municípios. A Figura 1 apresenta o mapa do Estado de Santa Catarina com a estimativa da população em 2015, em que as cores variam pela densidade regional, sendo as de menor população as mais claras; para maior população, as mais escuras; e os pontos em amarelos são os Institutos Federais espalhados pelo Estado. A obtenção dos arquivos em formato shape para a construção dos mapas foi obtida no site do IBGE (2015b). Para a construção do mapa temático, foi utilizada a medida separatriz dos quintis. A série é ordenada em cinco partes e cada uma contém $20 \%$ de seus elementos. Este cálculo foi feito automaticamente pelo pacote maptools.

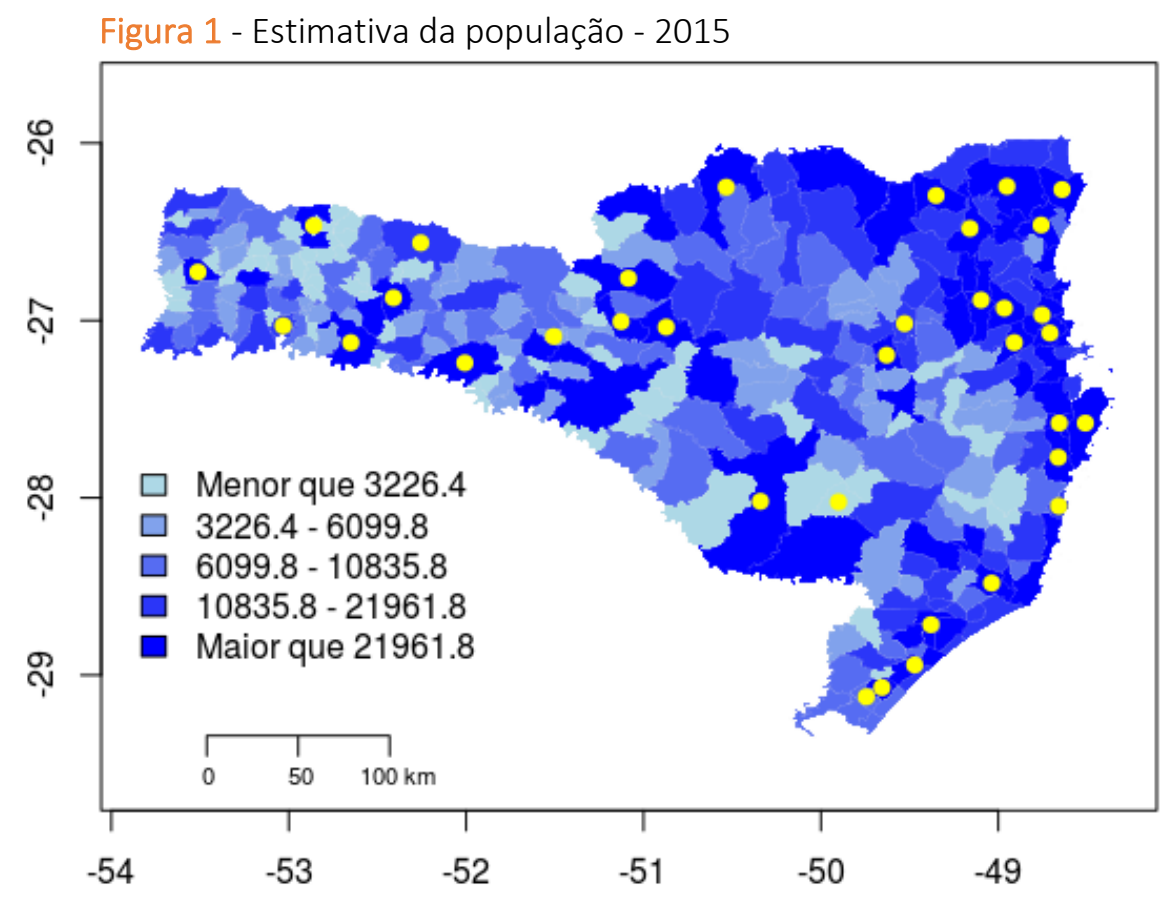

Fonte: Elaborado pelos autores.

Aplicando o algoritmo das p-medianas para localização de duas novas unidades, pode-se observar, na Figura 2, as cidades selecionadas com pontos vermelhos: Curitibanos e Mafra, assim como as cidades atendidas por cada Instituto. Curitibanos atenderia a 14 cidades, totalizando uma população atendida de 115.787 pessoas. Mafra atenderia a quatro cidades, com uma população atendida de 104.233 pessoas. Os resultados completos podem ser observados na Tabela 1. 


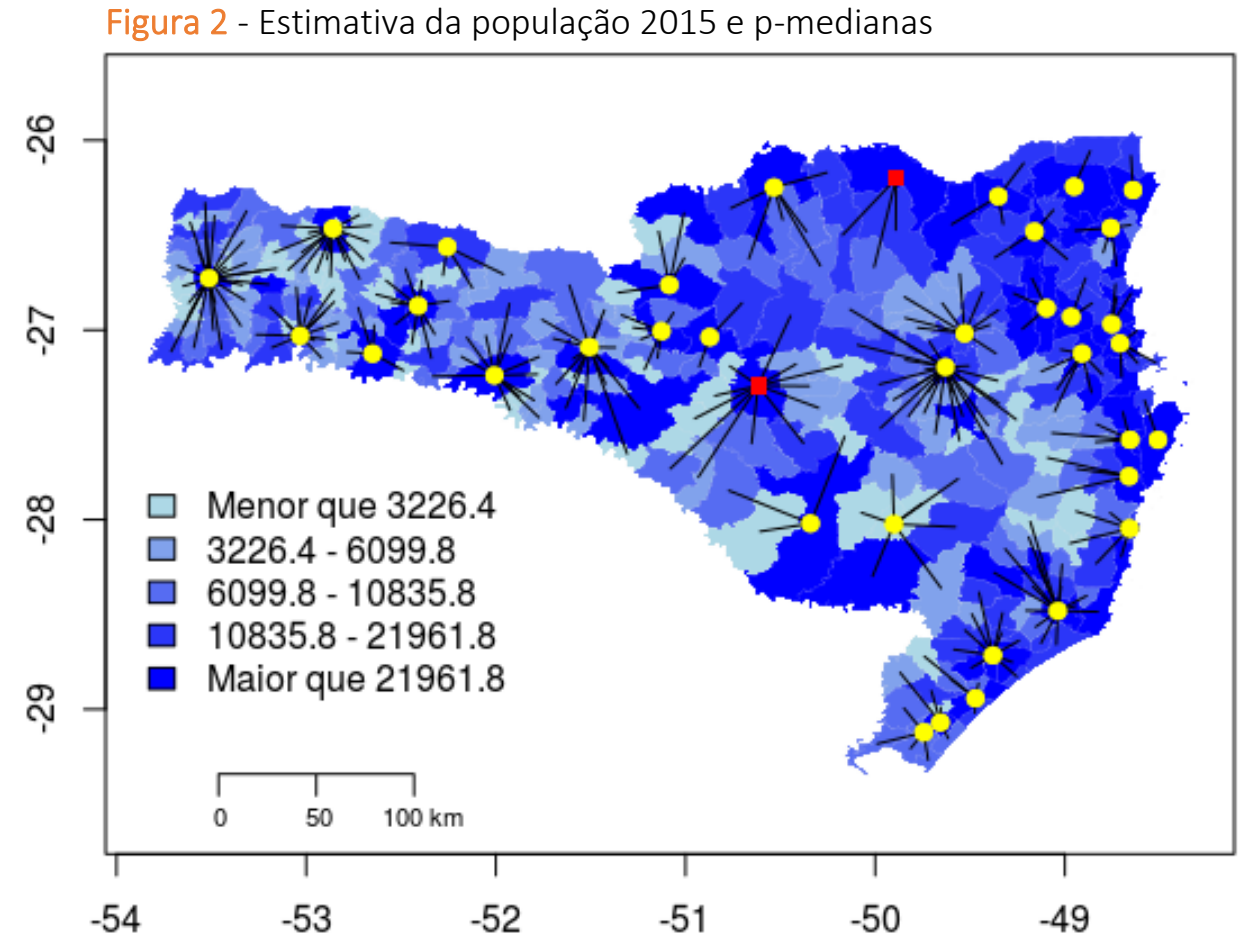

Fonte: Elaborado pelos autores.

Tabela 1 - Quantidade de Cidades Atendidas (Alocadas) e População Estimada Atendidas - 2015 Continua

\begin{tabular}{|c|c|c|}
\hline Cidades & Alocados & População \\
\hline Lages & 4 & 171.220 \\
\hline Mafra* & 4 & 104.233 \\
\hline Gaspar & 3 & 90.425 \\
\hline Itajaí & 4 & 328.153 \\
\hline Brusque & 7 & 210.690 \\
\hline Caçador & 5 & 118.597 \\
\hline Chapecó & 5 & 226.556 \\
\hline Ibirama & 10 & 80.823 \\
\hline Luzerna & 16 & 171.514 \\
\hline Palhoça & 5 & 191.968 \\
\hline Sombrio & 5 & 58.818 \\
\hline Tubarão & 16 & 322.328 \\
\hline Urupema & 8 & 62.365 \\
\hline Videira & 7 & 77.738 \\
\hline Xanxerê & 11 & 127.251 \\
\hline Araquari & 5 & 113.168 \\
\hline Blumenau & 4 & 474.829 \\
\hline Camboriú & 6 & 331.746 \\
\hline Criciúma & 11 & 398.519 \\
\hline Garopaba & 6 & 88.533 \\
\hline São José & 5 & 315.341 \\
\hline Araranguá & 5 & 94.335 \\
\hline Canoinhas & 7 & 114.376 \\
\hline
\end{tabular}


Conclusão Tabela 1

\begin{tabular}{l|rc}
\hline \multicolumn{1}{c|}{ Cidades } & Alocados & População \\
\hline Concórdia & 13 & 136.497 \\
Fraiburgo & 3 & 57.742 \\
Joinville & 24 & 578.937 \\
Rio do sul & 15 & 271.201 \\
São Carlos & 14 & 106.149 \\
Curitibanos* & 5 & 115.787 \\
Abelardo Luz & 2 & 37.400 \\
Florianópolis & 4 & 483.634 \\
Jaraguá do Sul & 4 & 209.743 \\
São Bento do Sul & 5 & 149.662 \\
Santa Rosa do Sul & 26 & 41.425 \\
São Miguel do Oeste & 2 & 213.020 \\
São Francisco do Sul & 17 & 66.743 \\
São Lourenço do Oeste & 295 & 77.724 \\
TOTAL & & 6.819 .190 \\
\hline
\end{tabular}

Fonte: Elaborado pelos autores.

*Novas Localizações

\subsection{Cenário 2 - Formandos nos Ensinos Fundamental e Médio}

Os Institutos Federais oferecem várias modalidades de cursos, das quais $50 \%$ das vagas ofertadas são de Ensino Médio e 20\% para formação de formadores. Para os cursos técnicos integrados ao Ensino Médio, são exigidos o Ensino Fundamental completo, e para os cursos técnicos concomitantes (simultâneos) ao Ensino Médio, é exigida a 1ao ou a 2ạ série do Ensino Médio completa (dependendo do curso). Já para os cursos técnicos subsequentes, é exigido o Ensino Médio completo. Os cursos superiores oferecidos pelos Institutos são os cursos de bacharelado, licenciatura e tecnologia, que exigem formação completa no Ensino Médio.

Desta forma, a escolha desta variável, número de formandos no Ensino Fundamental e Médio é um fator importante para a tomada de decisão, apesar de que, no mapa de população e no mapa de formandos (Figura 3), observa-se praticamente a mesma solução. Isso se justifica pelo fato de que o número de formandos está relacionado com o número de habitantes da cidade. 
Figura 3 - Formandos no Ensino Fundamental e Médio 2012

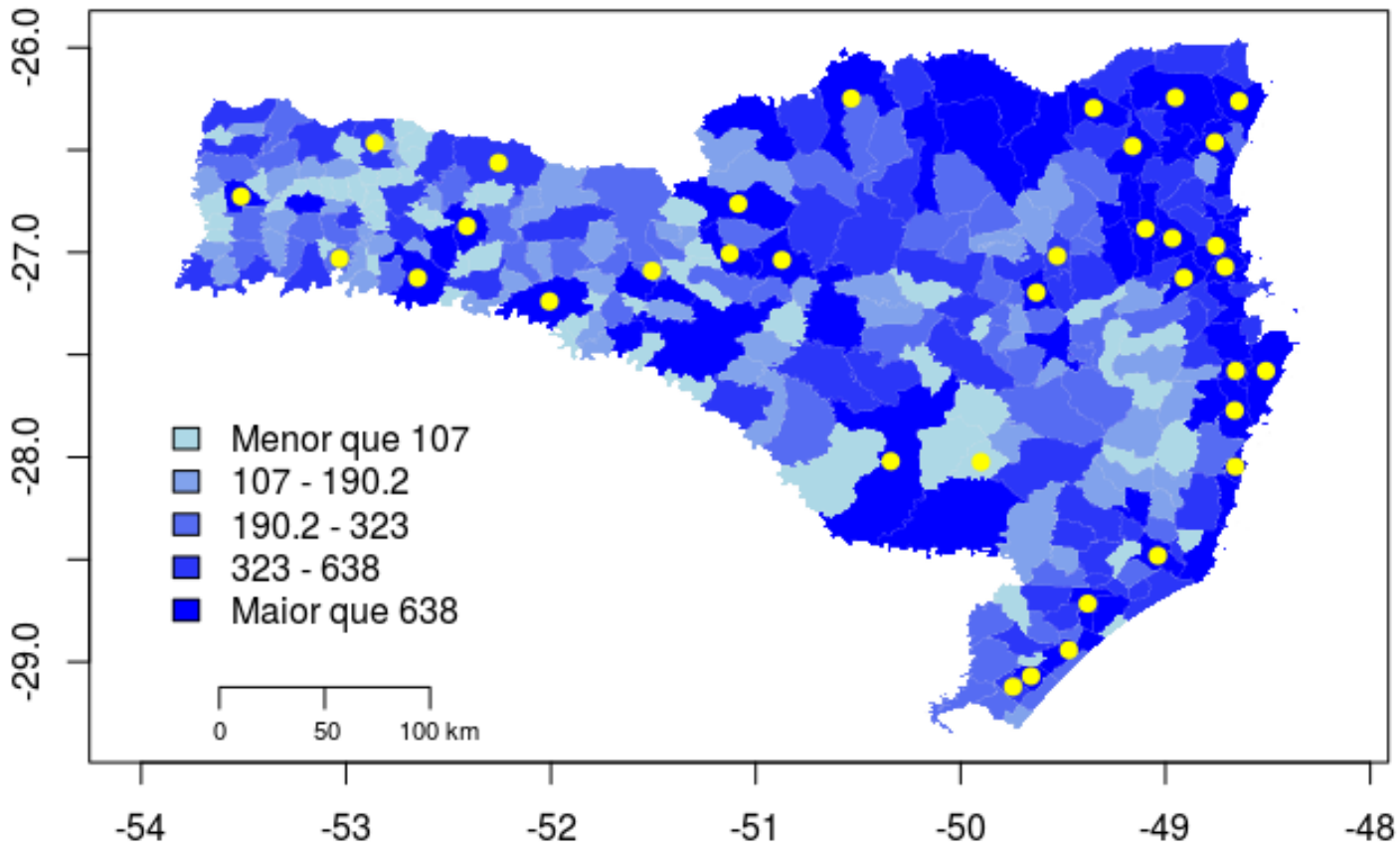

Fonte: Elaborado pelos autores.

Deste modo, aplicando o algoritmo das $p$-medianas para localização de duas novas unidades, obtém-se o resultado observado na Figura 4, isto é, as cidades selecionadas com pontos vermelhos são novamente Curitibanos e Mafra, assim como as respectivas cidades atendidas por cada Instituto.

Figura 4 - Formandos no Ensino Fundamental e Médio e p-medianas

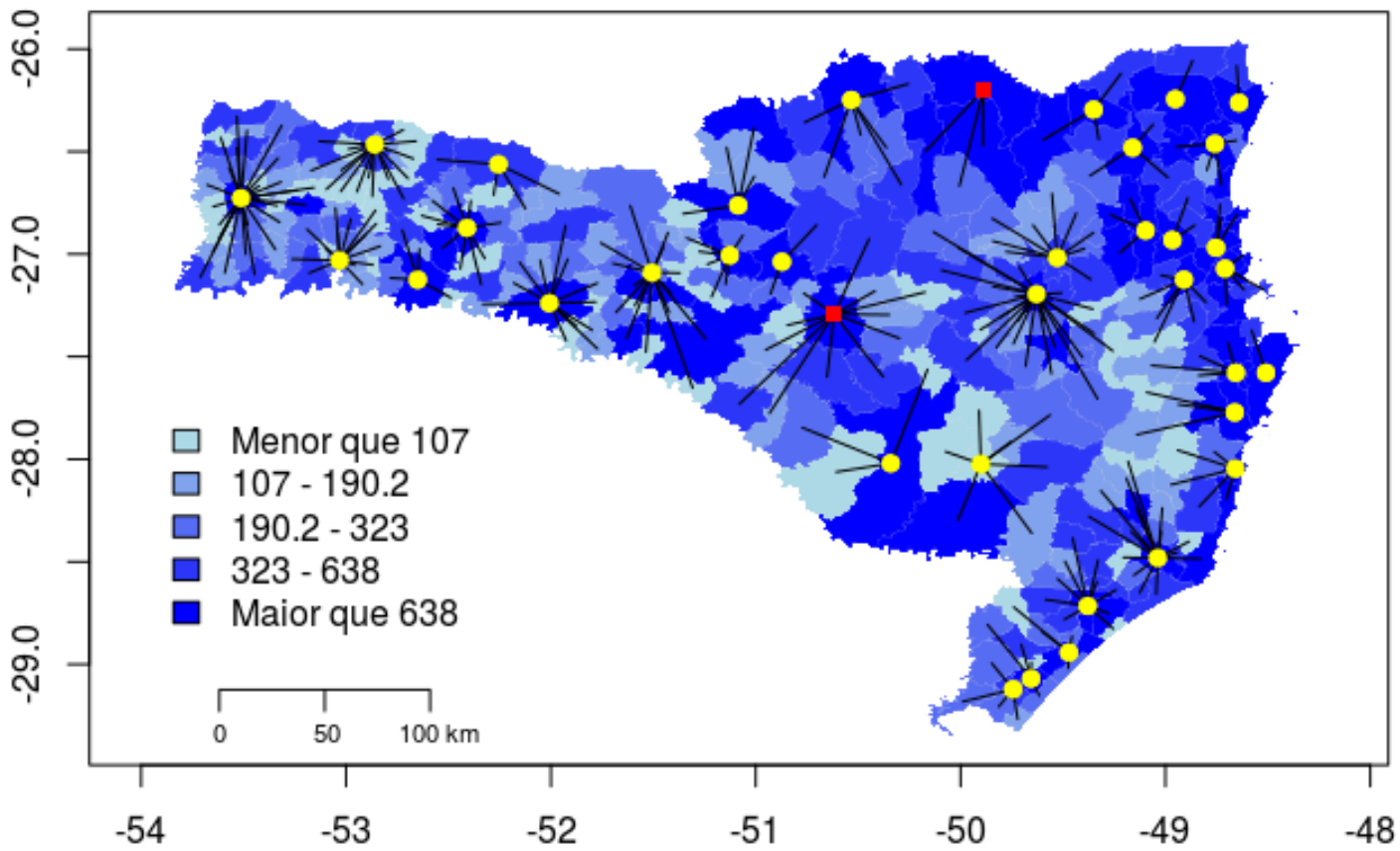


Curitibanos atenderia 14 cidades, do mesmo modo que o resultado anterior para a população, totalizando uma população de formandos atendida de 4.270 estudantes. Mafra atenderia quatro cidades, igual ao resultado anterior para a população, totalizando a população de formandos atendida de 3.623 estudantes. Maiores detalhes estão descritos na Tabela 2.

Tabela 2- Quantidade de Cidades Atendidas (alocadas) e Formandos do Ensino Fundamental e Ensino Médio Atendidas (população)- 2012

\begin{tabular}{|c|c|c|}
\hline Cidades & Alocados & População \\
\hline Lages & 4 & 5739 \\
\hline Mafra* & 4 & 3623 \\
\hline Gaspar & 3 & 2317 \\
\hline Itajaí & 4 & 8057 \\
\hline Brusque & 7 & 4898 \\
\hline Caçador & 5 & 3934 \\
\hline Chapecó & 5 & 6013 \\
\hline Ibirama & 10 & 2369 \\
\hline Luzerna & 16 & 5224 \\
\hline Palhoça & 5 & 5243 \\
\hline Sombrio & 5 & 1808 \\
\hline Tubarão & 16 & 9219 \\
\hline Urupema & 8 & 1880 \\
\hline Videira & 7 & 2309 \\
\hline Xanxerê & 11 & 4051 \\
\hline Araquari & 5 & 3160 \\
\hline Blumenau & 4 & 11347 \\
\hline Camboriú & 6 & 7557 \\
\hline Criciúma & 11 & 10886 \\
\hline Garopaba & 6 & 2737 \\
\hline São José & 5 & 8285 \\
\hline Araranguá & 5 & 2795 \\
\hline Canoinhas & 7 & 3966 \\
\hline Concórdia & 13 & 4055 \\
\hline Fraiburgo & 3 & 1802 \\
\hline Joinville & 2 & 14558 \\
\hline Rio do sul & 24 & 8259 \\
\hline São Carlos & 15 & 3203 \\
\hline Curitibanos* & 14 & 4270 \\
\hline Abelardo Luz & 5 & 1301 \\
\hline Florianópolis & 2 & 11816 \\
\hline Jaraguá do Sul & 4 & 5364 \\
\hline São Bento do Sul & 4 & 4144 \\
\hline Santa Rosa do Sul & 5 & 1364 \\
\hline São Miguel do Oeste & 26 & 6785 \\
\hline São Francisco do Sul & 2 & 1705 \\
\hline São Lourenço do Oeste & 17 & 2633 \\
\hline TOTAL & 295 & 188.676 \\
\hline
\end{tabular}

Fonte: Elaborado pelos autores. ${ }^{*}$ Novas Localizações 


\subsection{Cenário 3 - Analfabetismo}

Conforme Infante (1994), a UNESCO define que: "uma pessoa funcionalmente analfabeta é aquela que não pode participar de todas as atividades nas quais a alfabetização é requerida para uma atuação eficaz em seu grupo e comunidade, e que lhe permitem, também, continuar usando a leitura, a escrita e o cálculo a serviço do seu próprio desenvolvimento e do desenvolvimento de sua comunidade". (Infante, 1994, p. 7).

É importante destacar que, para uma ação efetiva, a alfabetização deve estar integrada a uma política de educação de jovens e adultos, para que os estudantes deem continuidade ao seu processo educacional.

Para este cenário, foi utilizada a taxa de analfabetismo, e conforme pode ser verificado na Figura 5, as localidades onde ocorrem as maiores taxas de analfabetismo são as cidades que possuem menor população.

Figura 5 - Analfabetismo em 2010

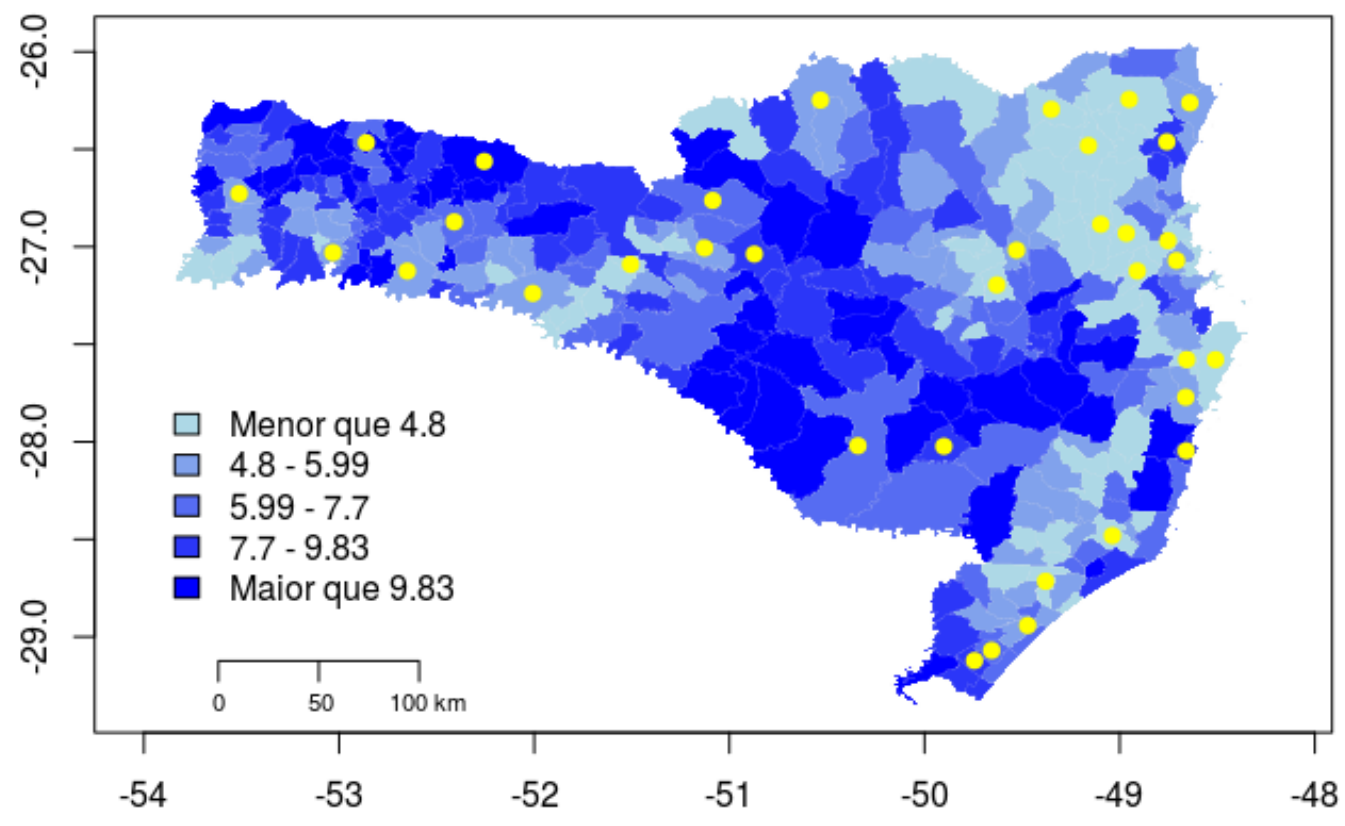

Fonte: Elaborado pelos autores.

Aplicando o algoritmo das $p$-medianas para localização de duas novas unidades, obteve-se que as cidades selecionadas com pontos vermelhos são Cerro Negro e São Cristóvão do Sul, assim como as respectivas cidades atendidas por cada Instituto (Figura 6).

A cidade de Cerro Negro atenderia sete cidades, cuja taxa média de analfabetismo da região é de $12,98 \%$, sendo a taxa de analfabetismo de Cerro Negro é de 17,07\%, a maior do estado. Já São Cristóvão do Sul atenderia 11 cidades, com taxa média de analfabetismo da região de 9,55\%, e taxa de analfabetismo da Cidade de São Cristóvão do Sul de $12,82 \%$. Os resultados completos podem ser 
observados na Tabela 3.

Figura 6 - Analfabetismo 2015 e $p$-medianas

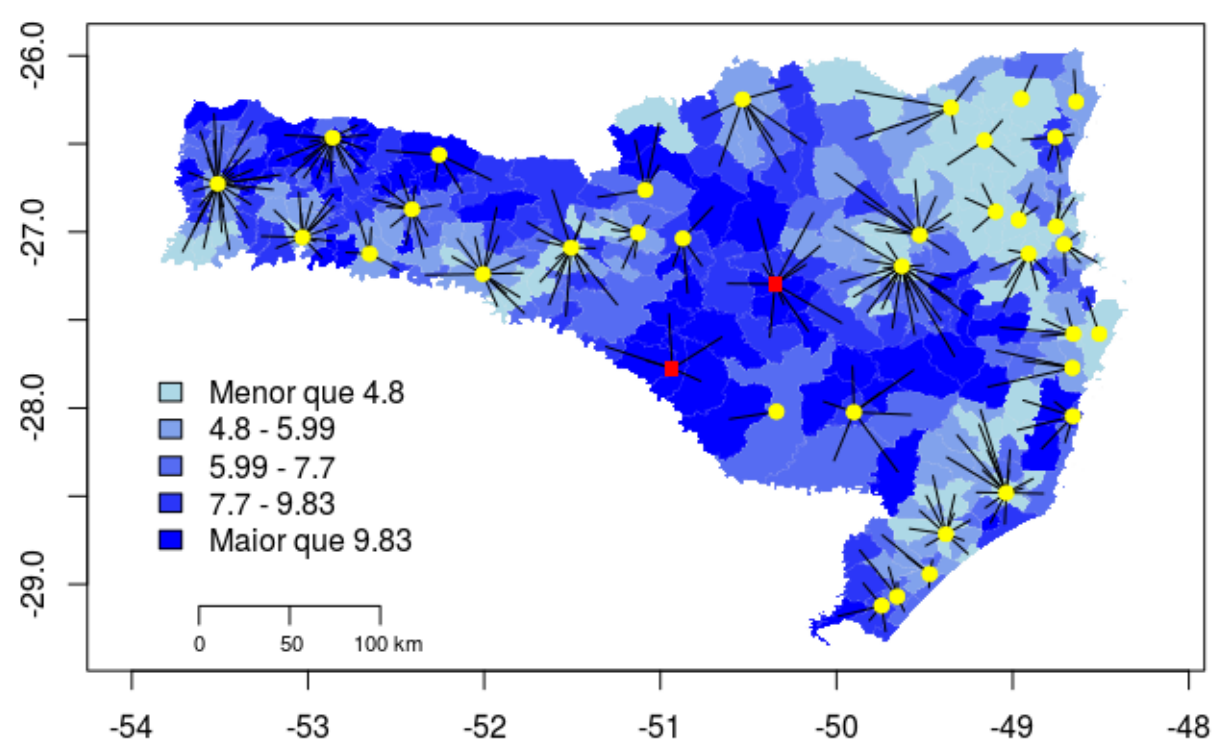

Fonte: Elaborado pelos autores.

Tabela 3- Quantidade de Cidades Atendidas (Alocadas) e Taxa de Analfabetismo Médio - 2010-Continua

\begin{tabular}{l|c|c}
\hline & Cidades & Taxa média \\
\hline Lages & 2 & 8,13 \\
Gaspar & 3 & 5,07 \\
Itajaí & 4 & 5,31 \\
Brusque & 7 & 5,86 \\
Caçador & 5 & 8,73 \\
Chapecó & 5 & 7,69 \\
Ibirama & 11 & 5,85 \\
Luzerna & 15 & 6,56 \\
Palhoça & 5 & 7,26 \\
Sombrio & 5 & 6,97 \\
Tubarão & 16 & 5,63 \\
Urupema & 8 & 9,94 \\
Videira & 7 & 5,66 \\
Xanxerê & 11 & 8,51 \\
Araquari & 5 & 6,28 \\
Blumenau & 4 & 3,45 \\
Camboriú & 6 & 5,04 \\
Criciúma & 11 & 4,29 \\
Garopaba & 6 & 8,19 \\
São José & 5 & 6,29 \\
Araranguá & 5 & 6,02 \\
Canoinhas & 5 & 7,67
\end{tabular}


Conclusão Tabela 3

\begin{tabular}{l|r|r} 
& & Taxa média \\
\hline Concórdia & Alocados & 5,81 \\
Fraiburgo & 13 & 11,16 \\
Joinville & 5 & 5,57 \\
Rio do Sul & 2 & 6,9 \\
São Carlos & 21 & 7,67 \\
Cerro Negro* & 15 & 12,98 \\
Abelardo Luz & 7 & 10,17 \\
Florianópolis & 5 & 5,71 \\
Jaraguá do Sul & 2 & 3,62 \\
São Bento do Sul & 4 & 5,03 \\
Santa Rosa do Sul & 6 & 8,87 \\
São Miguel do Oeste & 5 & 7,93 \\
São Cristóvão do Sul* & 26 & 9,55 \\
São Francisco do Sul & 11 & 5,38 \\
São Lourenço do Oeste & 2 & 10,44 \\
TOTAL & 17 & 7,29 \\
\hline
\end{tabular}

Fonte: Elaborado pelos autores.

*Novas Localizações

\section{Considerações finais}

O modelo matemático de solução exata demonstrou ser de difícil aplicação quando se tem grande número de variáveis, pois a solução se torna inviável computacionalmente. A aplicação proposta nesse caso é a heurística Teiz e Bart para localização de p-medianas.

As heurísticas Teiz e Bart apresentaram resultados satisfatórios para a determinação de soluções para o problema das p-medianas, indicando, desta forma, onde estão as melhores localizações para se implantar os Institutos Federais. A partir da heurística, foram obtidas as seguintes cidades: Curitibanos, Mafra, Cerro Negro e São Cristóvão do Sul. Desta forma, é possível para o gestor da organização avaliar melhor as informações referentes à instalação dos institutos nas respectivas cidades. Cabe ressaltar que, como se trata de Institutos Federais, há várias questões envolvidas, sejam políticas e/ou técnicas, que podem interferir na decisão de instalar-se ou não o respectivo instituto.

Nos cenários aplicados, os resultados dependem da demanda. Logo, pode-se verificar que a quantidade de população e a quantidade de formandos têm grandezas proporcionais, ou seja, cidades com maior número de habitantes têm uma quantidade maior de formandos, e uma cidade com pouca população tem uma pequena quantidade de formandos.

Uma das limitações do trabalho é o fato das distâncias entre uma cidade para outra terem sido calculadas como distâncias euclidianas. Então, as distâncias apresentadas são apenas uma aproximação das distâncias reais. Da mesma forma, o centróide da área não coincide com o centróide da distribuição populacional. Caso fossem utilizadas as distâncias reais, haveria uma melhor precisão do resultado. Para 
um próximo trabalho, sugere-se utilizar distâncias reais de uma cidade para outra.

Para localização de novas unidades de ensino dos Institutos Federais e melhorar a tomada de decisão, sugere-se também localizar unidades de outras instituições, como, por exemplo, campus da Universidade Federal de Santa Catarina (UFSC) e Universidade do Estado de Santa Catarina (UDESC), para ofertas de cursos superiores e Escolas de Ensino Técnico para ofertas de cursos técnicos, bem como avaliar as instituições privadas localizadas na região.

\section{Referências}

Barros, A. J. S., \& Lehfeld, N. A. S. (2000). Fundamentos de Metodologia: um Guia para a Iniciação Científica. São Paulo: Makron Books.

Bivand, R. et al. (2018). Package maptools: Tools for Handling Spatial Objects. Version 0.9-4. Disponível em : https://cran.r-project.org/web/packages/maptools/index.html. Acesso em: 10 dezembro de 2018.

Brunsdon, C. (2015) Package tbart: Teitz and Bart's p-Median Algorithm. Version 1.0. Disponível em: https://cran.r-project.org/web/packages/tbart/index.html. Acesso em: 08 novembro de 2018.

Christofides, N. (1975). Graph theory: an algorithmic approch. New York: Academic Press Inc.

Daskin, M. S. (1995). Network and Discrete Location - Models, Algorithms and Aplications. New York: John Wiley \& Sons.

Calache, L. D. D. R., \& Camargo, V. C. B. (2016). Proposta de um método de localização e alocação de pontos de coleta seletiva. Revista Eletrônica Pesquisa Operacional para o Desenvolvimento, 8(2), 137-162.

Camara, M. V. O., Ribeiro, G. M., Quadros, S. G. R., Hora, C. A. H., Nascimento, C. J. F., \& Abramides, C. A. (2016). Modelagem matemática aplicada no planejamento de pesquisa de tráfego rodoviário. Revista Produção Online, 16(2), 722-742. https://doi.org/10.14488/1676-1901.v16i2.2125

Garcia, A. E. N. (2015). Aplicação da p-Mediana na Localização da Coordenadoria de Educação da Regional do Médio Paraíba do Estado do Rio. Dissertação de mestrado, Universidade Federal Fluminense, Rio de Janeiro.

Gil, A. C. (2017). Como Elaborar Projetos de Pesquisa. São Paulo: Atlas.

Goldbarg, M. C., \& Luna, H. P. L. (2000). Otimização Combinatória e Programação linear: Modelos e Algoritmos. Rio de Janeiro: Campus.

Goudard, B., Oliveira, F. H., \& Gerente, J. (2015). Avaliação de modelos de localização para análise da distribuição espacial de unidades básicas de saúde. Revista Brasileira de Cartografia, Sociedade Brasileira de Cartografia, Geodésia, Fotogrametria e Sensoriamento Remoto, 67(1), 15-34. ISSN: 1808-0936.

Hakimi, S. L. (1965). Optimum Distribution of Switching Centers in a Communication Network and Some Related Graph Theoretic Problems. Operations Research, 13, 462-475. https://doi.org/10.1287/opre.13.3.462.

Hakimi, S. L. (1964). Optimum Location of Switching Centers and the Absolute Centers and the 
Medians of a Graph. Operations Research, 12, 450-459. https://www.jstor.org/stable/168125. Horner, D. (2009). Resolução do Problema das P-medianas não Capacitadas: comparação de Algumas Técnicas Heurísticas. Dissertação de mestrado, Universidade Federal de Santa Catarina, Florianópolis.

Instituto Brasileiro de Geografia e Estatística (2011). Censo Demográfico 2010. Disponível em: http://www.censo2010.ibge.gov.br/. Acesso em: 05 de outubro de 2018

Instituto Brasileiro de Geografia e Estatística (2015a,). Estimativas Populacionais para os Municípios e para as Unidades da Federação Brasileiros. Disponível em:

https://ww2.ibge.gov.br/home/estatistica/populacao/estimativa2015/estimativa_dou.shtm. Acesso em: 08 de outubro de 2018.

Instituto Brasileiro de Geografia e Estatística (2015b). Índice de Organização do Território Malhas Territoriais. Disponível em:

ftp://geoftp.ibge.gov.br/organizacao_do_territorio/malhas_territoriais/malhas_municipais/municipi o_2015/UFs/SC/. Acesso em: 20 de outubro de 2018.

Instituto Federal Catarinense (2018). Relatório de Gestão do Exercício de 2017. Disponível em: http://ifc.edu.br/wp-content/uploads/2018/07/Relatório-de-Gestão-2017.pdf. Acesso em: 30 de outubro de 2018.

Instituto Nacional de Estudos e Pesquisas Educacionais Anísio Teixeira (2012). Microdados para download. Disponível em: http://inep.gov.br/microdados. Acesso em: 10 de novembro de 2018.

Infante, M. I. (1994). Investigación regional sobre analfabetismo funcional. Santiago: UNESCO/OREALC.

Larson, R. C., \& Odoni, A. R. (1981). Urban Operations Research. New Jersey: Prentice-Hall.

Pizzolato, N. D., Raupp, F. M. P., \& Alzamora, G. S. (2012). Revisão de Desafios Aplicados em Localização com Base em Modelos da p-mediana e suas Variantes. Pesquisa Operacional para o Desenvolvimento, 4(1), 13-42. Disponível em: https://www.podesenvolvimento.org.br/podesenvolvimento/article/view/174/161. Acesso em: 12 de novembro de 2018.

R Core Team. (2018). R: A language and environment for statistical computing. R Foundation for Statistical Computing. Disponível em: http://www.R-project.org/. Acesso em: 12 de outubro de 2018.

Randhawa, S. U., \& West, T. M. (1995). An Integrated Approach to Facility Location Problems. Computers \& Industrial Engineering, 29(1-4), 261-265. https://doi.org/10.1016/03608352(95)00082-C.

Romero, B. C. (2006). Análise da Localização de Plataformas Logísticas: uma aplicação ao caso da ETSP - Entreposto Terminal São Paulo - da CEAGESP. Dissertação de mestrado, Universidade de São Paulo, São Paulo.

Silva. R. T. (2009). Avaliação da Distribuição Espacial de Escolas a Partir de um Modelo Não Capacitado com Auxílio da Ferramenta Sig: Um Estudo de Caso da Cidade de Volta Redonda-RJ. Dissertação de mestrado, Universidade Federal Fluminense, Volta Redonda.

Teitz, M. B., \& BART, P. (1968). Heuristics Methods for Estimating the Generalized Vertex Median of a Weighted Graph. Operations Research Society, 16(5), 901-1091.

https://doi.org/10.1287/opre.16.5.955. 